\title{
Histological Confirmation of Bypass Tracts for Atrioventricular Conduction Originating from the Right Atrium
}

\author{
Toyomi Sano, M.D., Fumio Suzuri, M.D., \\ and Hiromichi Tsuchmashi, M.D.
}

\section{SUMmARY}

Histological determination was made on the preparations from the rabbit heart presurned to be bypass tracts by the previous functional study. As the first bypass tract a muscle strip composed of the apparently ordinary cardiac muscle was found to leave the right atrium and reach the bundle of $\mathrm{His}$ at or near the bifurcation, bypassing the $\mathrm{A}-\mathrm{V}$ node. As the second bypass tract a bundle of apparently ordinary cardiac muscle fibers leaves the lateral wall of the right atrium above the $A-V$ groove, descending and reaching the lateral wall of the right ventricle. A part of the third bypass tract was found below the right atrium close to the interatrial septum and its terminal part was found to enter the left side of the A-V node anterior to the ordinary atrio-nodal connection. Since its middle part could not be differentiated clearly from the ordinary atrial muscle fibers, the histological confirmation is not considered to be complete as yet, as far as the third bypass tract is concerned.

These histological findings explain the short P-R interval and the almost normal ventricular complex of the first bypass conduction, the short P-R interval and the abnormal configuration of the ventricular complex of the second bypass conduction, and the long $\mathrm{P}-\mathrm{R}$ interval and normal ventricular complex of the third bypass conduction. As additional functional findings the short $P-R$ interval of the first bypass was found to show relatively constant values in spite of the change in the stimulus frequency and the longer than normal P-R interval of the third bypass to decrease at first and increase markedly later as the stimulus frequency was increased.

\section{Additional Indexing Words :}

The bundle of Kent Wolff-Parkinson-White syndrome The A-V bypass tract studied anatomically by James Acetylcholine Suction electrode Microelectrode Rate-dependent A-V conduction time Network of the A-V node The bundle of His Rabbit heart

$7 \mathrm{HE}$ discovery by Kent ${ }^{1-7)}$ of the lateral atrio-ventricular junction is almost as old as that by $\mathrm{His}^{81}$ and Tawara ${ }^{9)}$ of the specific atrio-ventricular

From the Institute for Cardiovascular Diseases, Tokyo Medical and Dental University, Tokyo. Received for publication December 4, 1970. 
conduction system. The first ascription of the so-called Wolff-ParkinsonWhite syndrome to the bundle of Kent was also 40 years ago. ${ }^{10}$ In spite of such a long history it is only a few years ago that the function of the bundle of Kent is beginning to be established by various functional studies by Durrer et al., ${ }^{11)}$ Burchell ct al. ${ }^{12)}$ and Cobb et al. ${ }^{13}$ in the human open chest. As for the other bypass tracts of the A-V conduction, there have been some anatomical reports by Mahaim ${ }^{14-18)}$ and James. ${ }^{191,20)}$ Nothing can be said as to the clinical significance of these tracts because of the scantiness of the functional studies on them.

Independently from these anatomical studies, we have continued with our functional study on the conduction system of the heart and found some functional evidence for the 3 bypass tracts for the A-V conduction, which was reported elsewhere. ${ }^{21)}$ In this report are described the histological determination of such bypass tracts and additional functional findings.

\section{METHODS}

Most of the preparations, in which some evidences of the presence of some of the A-V bypass tracts were found by the functional studies, were examined histologically. Serial sections were made of the important regions. Van Gieson's stain was usually employed.

For functional studies the rabbit heart was isolated, perfused with aerated Tyrode solution and placed in the center of a plexiglass chamber containing about $70 \mathrm{ml}$. of the aerated Tyrode solution. Three electrodes were mounted on its 3 corners to obtain the lead II or III electrocardiogram. A suction electrode was placed in the center of the right ventricle to obtain its action potential. Change of conduction was examined by measuring the interval from the stimulus artifact to the beginning of the QRS complex (the S-R interval), when the frequency of the electrical stimulation applied on the outer wall of the right atrium was changed.

To determine the mode of conduction, the atrial and ventricular septa were exposed by a transverse incision below the $\mathrm{A}-\mathrm{V}$ groove. One microelectrode was inserted into the A-V node and another into the bundle of His. One pair of electrodes of the adjacent bipolar lead was placed near the fossa ovalis and another on the free wall of the right ventricle.

\section{Results}

In the previous functional study the first bypass tract was presumed to be present in 4 of 21 experiments. This was mostly found in the following way: after checking that the stimulation of all the usual points on the outer wall of the right atrium induced conduction to the ventricle in the control experiments, acetylcholine was added to the Tyrode solution to the concentration of 1 to $10 \mu \mathrm{g} . / \mathrm{ml}$. and complete or incomplete A-V block was induced. After 


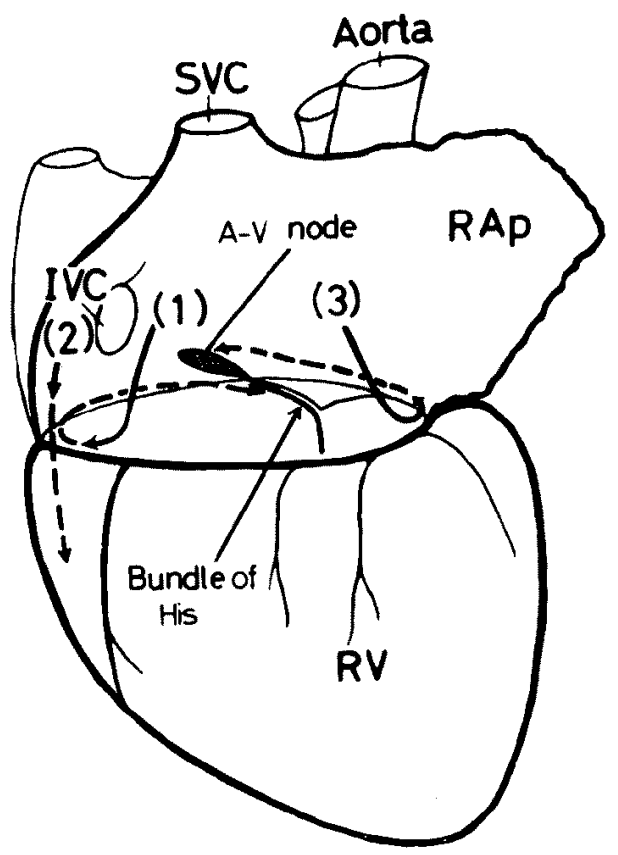

Fig. 1. Schematic diagram of the bypass tracts. (1) The first bypass tract with a short P-R interval and a normal ventricular complex. (2) The second bypass tract with a short $P-R$ interval and an abnormal ventricular complex. (3) The third bypass tract with a prolonged P-R interval and a normal ventricular complex.

$\mathrm{RAP}=$ right auricular appendage; $\mathrm{RV}=$ right ventricle; $\mathrm{SVC}=$ superior vena cava; IVG $=$ inferior vena cava.

this state an attempt was made to discover some point, the stimulation of which still induces conduction to the ventricle. Such a point was occasionally found anterior to the root of the inferior vena cava (point 1 in Fig. 1). In this conduction the S-R interval, which corresponds to the P-R interval, was shorter than in the control conduction and no significant alteration of the QRS-T configuration was noticed. The presence of the delta wave was not completely excluded because the stimulus artifact was too close to the ventricular complex. The experiments to localize the site of the abnormal conduction indicated it to be somewhere between the bundle of His and the ventricle, including both.

Rate-dependent change of the conduction time was peculiar by this bypass. In the control experiments the S-R interval first decreased slightly, but increased steadily when the stimulus frequency was increased gradually from 120 per min. (open circles in Fig. 2a). By a lower frequency of stimulation than this the atrium did not follow it, because the spontaneous activity by the sinus impulse dominated it. When acetylocholine was added to the Tyrode solution to the concentration of $1 \mu \mathrm{g} . / \mathrm{ml}$, the sinus impulses were 

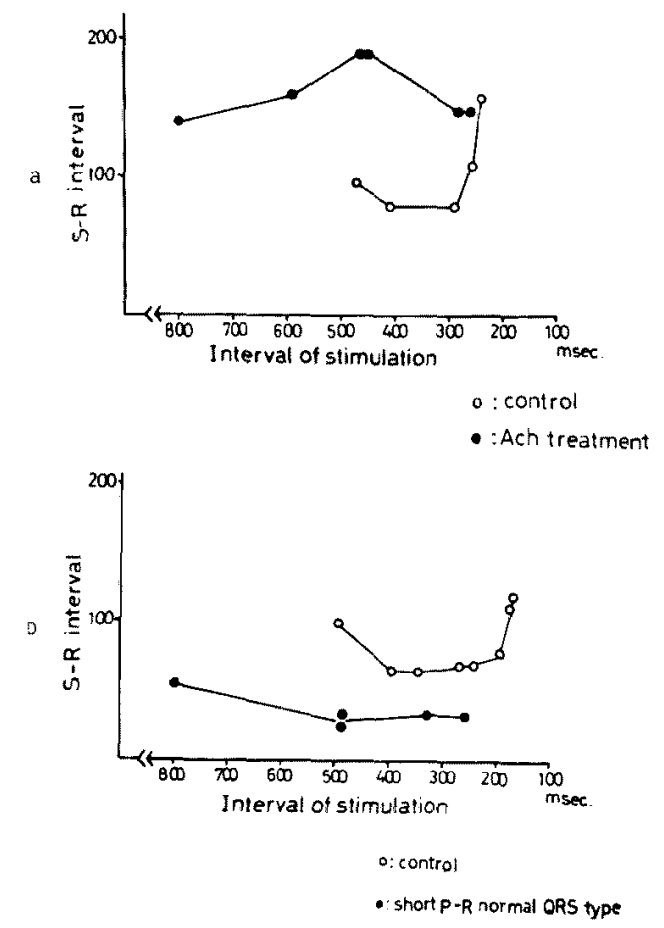

Fig. 2. Change of the A-V conduction time by the first bypass tract according to the change of stimulus frequency. Abscissae show the stimulation interval and ordinates show the S-R interval.

a: the result of a control experiment. Open circles show the S-R interval in the Tyrode solution as the control, and the closed circles when $1 \mu \mathrm{g} . / \mathrm{ml}$. of acetylcholine was added.

b: Open circles show the S-R interval of this preparation in the Tyrode solution as the control, and the closed circles that of the first bypass tract which was found after complete or incomplete A-V block by giving $5 / \mathrm{g} / \mathrm{ml}$. of acetylcholine.

The preparation does not follow the stimulus beyond a certain range of stimulus frequency. At a low frequency it does not follow the stimulus because the spontaneous activity takes over and also at a high frequency because the stimulus falls in the refractory period of the preceding beat.

suppressed and the atrium followed the stimulation by a far lower rate. The S-R interval through the His-Tawara conduction system was increased markedly (closed circles in Fig. 2a). In contrast, when the A-V conduction occurred through the first bypass tract, after the induction of complete or incomplete block of the His-Tawara system by acetylcholine, the S-R interval was decreased markedly and remained relatively constant with all the changes of the stimulus frequency (Fig. 2b).

Histological examination revealed that the apparently ordinary cardiac muscle left the right atrium and reached the bundle of His near the bifurcation, 


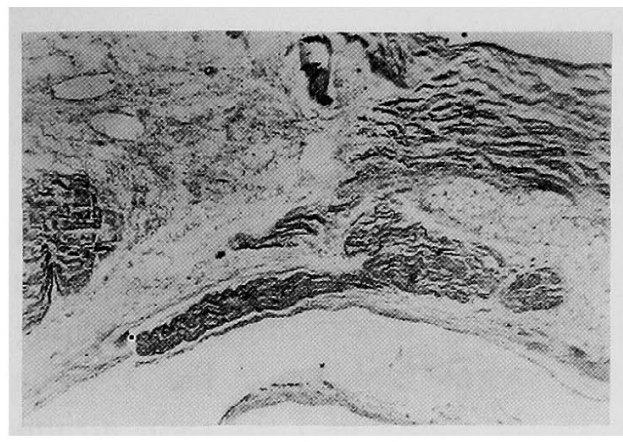

a

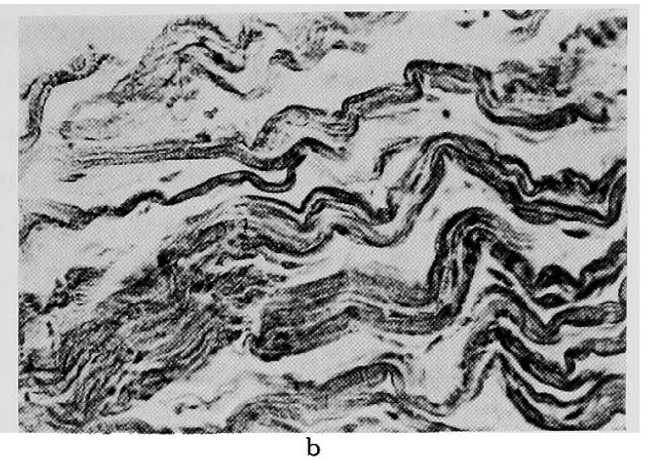

$\mathrm{b}$

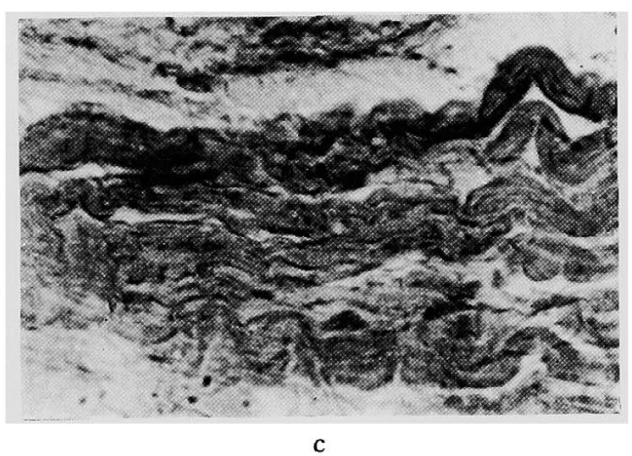

Fig. 3. Histological determination of the first bypass tract. Van Gieson's stain. Magnification, a: $\times 40 ; \mathrm{b}$ and $\mathrm{c}: \times 80$. See text for explanation.

bypassing the A-V node. An example is shown in Fig. 3. This preparation is one of the serial sections made every $10 \mu$. transverse-obliquely. In the upper part of Fig. 3a the right atrium is seen. A part of the right ventricle is seen on the left. In the lower central part is seen the bifurcation or the vicinity of the bifurcation of the bundle of His as a flat oval shape. A part of the right atrium is seen leaving and descending obliquely. This is seen reaching the bundlc of His at its right end in the figure. Fig. $3 \mathrm{~b}$ and $\mathrm{c}$ show larger magnification of the atrial part and the His part of this connection, respectively.

The second bypass tract was presumed to be present in 2 of 21 experiments. This was mostly found in the following way: in the complete or incomplete A-V block caused by the addition of acetylcholine another point was found to induce conduction to the ventricle by stimulation. This point was localized close to and postero-inferior to point 1 at the root of the inferior vena cava. In this stimulation the S-R interval was shortened and the QRS complex in the lead II electrocardiogram was inverted and widened, the latter being different from the stimulation of point 1 . When a transverse incision was made below this point, no response was observed. At this time 
the stimulation below this incision caused a ventricular response similar to that to the stimulation of point 2 .

Because of the relatively rare incidence of this type of bypass, the ratedependent change of its conduction time has not been determined as yet.

Histological examination revealed that in the right lateral wall around the A-V groove, a bundle of seemingly ordinary cardiac muscle fibers was found to leave the lateral wall of the right atrium, descending and reaching the lateral wall of the right ventricle. An example is shown in Fig. 4. The preparations are frontal serial sections. In the upper part the right atrium is seen and in the lower part the right ventricle is seen. A muscle strip, the appearance of which does not seem to be any different from that of the right

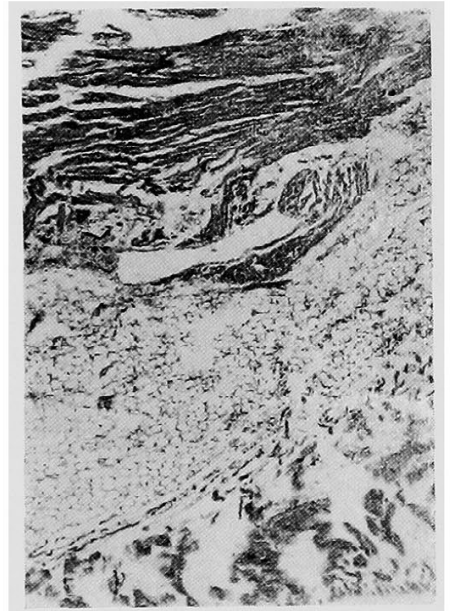

a

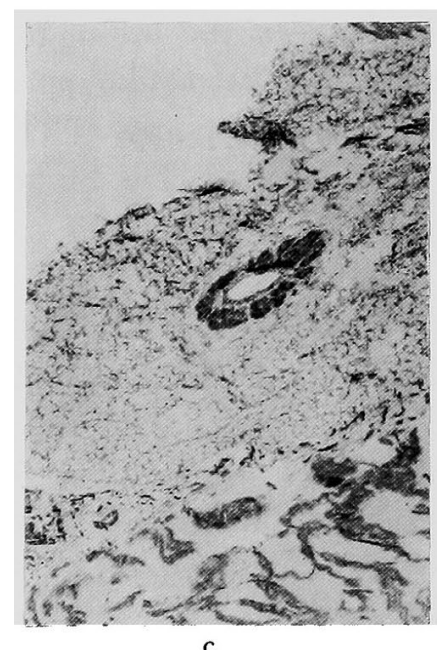

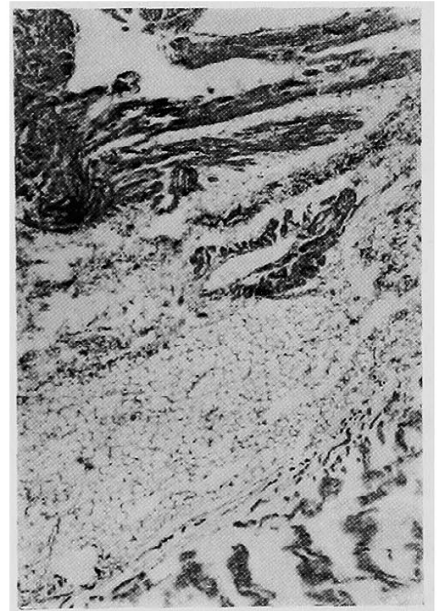

b

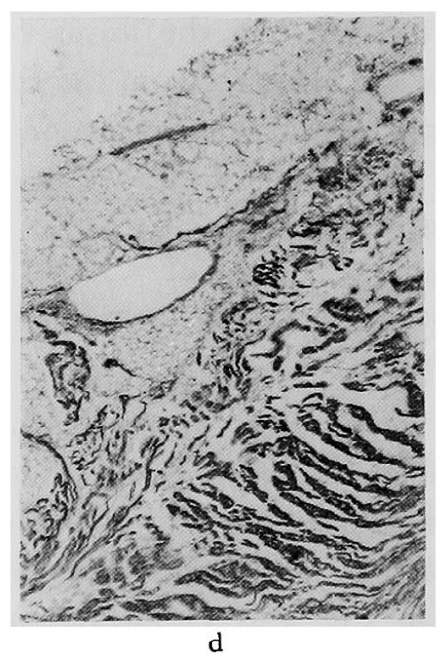

Fig. 4. Histological determination of the second bypass tract. Van Gieson's stain. Magnification, $\times 40$. See text for explanation. 
atrial muscle seen above, leaves the right atrium (Fig. 4a) descends obliquely surrounding a vessel (Figs. b and c) and reaches the right ventricle (Fig. d).

The third bypass tract was presumed to be present in 5 of 21 experiments. Its presence was first suspected without using acetylcholine, when the A-V

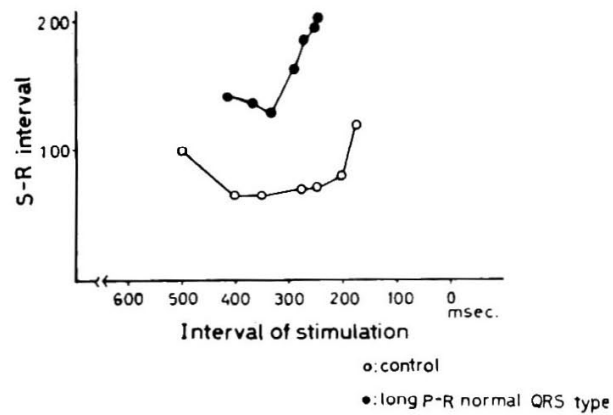

Fig. 5. Change of the A-V conduction time by the third bypass tract according to the change of stimulus frequency. The abscissa shows the stimulation interval and the ordinate the S-R interval. Open circles show the $\mathrm{S}-\mathrm{R}$ interval of this preparation in the Tyrode solution as the control and the closed circles that of the third bypass tract.

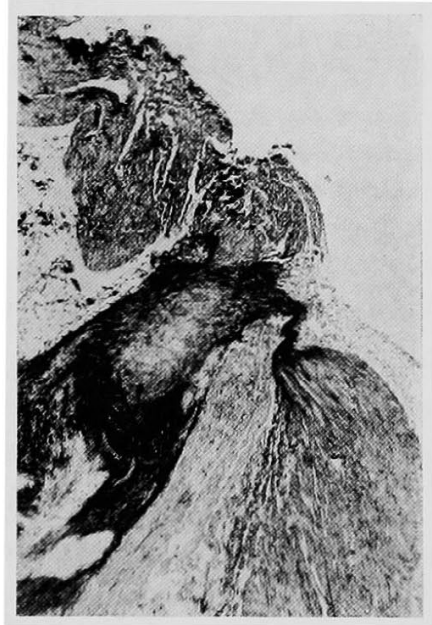

a

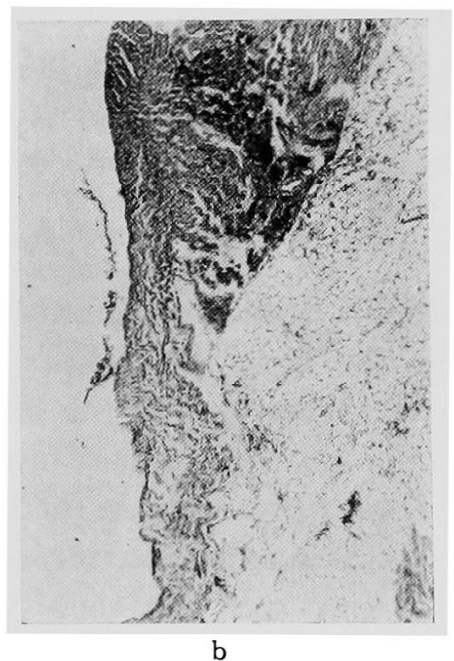

b

Fig. 6. Histological examination of the third bypass tract. Van Gieson's stain. Magnification, $\times 40$. The interatrial septum is seen in the center of the upper part of a. In the left upper corner a part of the right atrium is seen. A round bundle of the muscle fibers between them is relatively the first part of the third bypass tract. In the lower part the interventricular septum is seen on the right and the right bundle branch on the left.

In $b$ the atrioventricular node is seen on the left. A muscle mass which stained darker and has larger cells than the nodal ones enters the node from its left side. This is the end of the third bypass tract. 
conduction persisted with the prolongation of the conduction time and with the normal QRS-T pattern after all the normal routes of intra-atrial conduction pathways to the A-V node were incised. This persisting A-V conduction was blocked when the incision reached some anterior portion of the right atrium. The site of the abnormal conduction was localized by a different series of experiments exposing the atrial and ventricular septa. Stimulation of a point in the anterior wall of the right atrium above the incision just described, marked, as point 3 in Fig. 1, induced such a conduction with a longer $\mathrm{S}-\mathrm{R}$ interval and normal ventricular complex. The delay was found to be most conspicuous between the $\mathrm{A}-\mathrm{V}$ node and the bundle of His, but the interval between the bundle of $\mathrm{His}$ and the ventricle was also prolonged.

As the stimulus frequency was increased, the degree of the S-R prolongation decreased slightly at first and increased markedly thereafter (Fig. 5).

By the histological examination a part of the tract was found below the right atrium close to the interatrial septum (Fig. 6a) and its terminal part was found to enter the left side of the A-V node (Fig. 6b) anterior to the ordinary atrio-nodal connection. These parts showed histologically a transitional character between the specific nodal cells and ordinary myocardial fibers. However, the middle portion of this tract could not be clearly differentiated from the ordinary atrial muscle fibers. In this sense the histological confirmation is not considered to be complete as yet, as far as the third bypass tract is concerned.

\section{Discussion}

Histological findings explain the previous functional findings satisfactorily and support the deduction ${ }^{21}$ from them cited in Fig. 1. Entry into the bifurcation or the vicinity of the bifurcation of the bundle of His bypassing the A-V node and the ordinary myocardial appearance of the muscle fibers of the first bypass tract explain the short P-R interval and the almost normal ventricular complex of the electrocardiogram. Direct communication between the right atrium and the right ventricle with the apparently ordinary myocardial fibers of the second bypass tract bypassing the whole specialized $A-V$ conduction system explains the short P-R interval and the abnormal configuration of the ventricular complex. Abnormal entry into the A-V node of the third bypass tract is supposed to induce a detour within the network of the A-V node and the bundle of His, explaining the long P-R interval and the normal ventricular complex of the electrocardiogram. Whether or not the first tract is the same as that reported by James ${ }^{19), 20)}$ and the second tract as that reported by Kent ${ }^{1-7)}$ has not been determined as yet. 
To determine whether these bypass tracts are a normal structure or an anomaly of the rabbit heart requires a greater number of experiments. However, the rate of discovery of the first and third bypass tracts was too high to regard them as anomalies even with our small number of experiments. The reason why these bypass tracts do not operate under the normal sinus impulse spread is supposed to be due to the presence of some anatomic structure at some region that prevents easy conduction. At least in the usual states normal conduction through the His-Tawara A-V conduction system occurs more easily. Conduction may occur when the excitation wave comes from a different direction, for example in some instances when an atrial premature beat originates or when the shifting of the pacemaker occurs. In contrast the rate of discovery of the second bypass tract was low. This might be a kind of an anomaly.

\section{REFERENCES}

1. Kent, A. F. S. : J. Physiol. $14:$ XXIII, 1893.

2. Kent, A. F. S.: J. Physiol. $14: 233,1893$.

3. Kent, A. F. S. : Quart, J. Exper. Physiol. 7: 193, 1914.

4. Kent, A. F. S.: J. Physiol. $48:$ XXII, 1914.

5. Kent, A. F. S.: J. Physiol. 48 : LVII, 1914.

6. Kent, A. F. S. : J. Physiol. 48 : LXIII, 1914.

7. Kent, A. F. S. : Proc. Roy. Soc. 87: 198, 1913-1914.

8. His, W., Jr.: Arb. med. Klinik zu Leipzig, p. 14, 1893.

9. Tawara, S.: Das Reizleitungssystem des Säugetierherzens. Eine anatomisch-histologische Studies über das Atrioventrikularbündel und die Purkinjeschen Fäden. Fischer, Jena, 1906.

10. Wolf, L., Parkinson, J., and White, P. D. : Am. Heart J. 5 : 685, 1930.

11. Durrer, D. and Roos, J. P.: Girculation 35 : 15, 1967.

12. Burchell, H. B., Frye, R. L., Anderson, M. W., ct al.: Circulation $36: 663,1967$.

13. Cobb, F. R., Blumenschein, S. D., Sealy, W. C., et al. : Circulation 38 : 1018, 1968.

14. Mahaim, I. et Clerc, A.: C. R. Soc. Biol (Paris) $109: 183,1932$.

15. Mahaim, I. : Ann. Méd. 32 : 347, 1932.

16. Mahaim, I. et Benatt, A. : Cardiologia $1: 61,1937$.

17. Mahaim, I. et Winston, M. : Cardiologia 5: 189, 1941.

18. Mahaim, I. : Am. Heart J. 33: 651, 1947.

19. James, T. N. : Am. Heart J. $66: 498,1963$.

20. James, T. N.: Circulat. Res. $20: 638,1967$.

21. Sano, T., Suzuki, F, and Tsuchihashi, H. : Circulation $41: 413,1970$. 